\title{
Enrollment of Patients with Brain Metastases in Clinical Trials
}

\author{
Kosov M*, Dumpis T, Riefler J and Belotserkovskiy M \\ PSI CRO AG, Switzerland
}

Submission: September 27, 2017; Published: October 13, 2017

*Corresponding author: Maxim Kosov, PSI CRO AG (Zug, Switzerland), PSI Pharma Support America, 875 First Avenue, King of Prussia, PA 19406, USA; Tel: +1 215837 6222; +1 267464 2554; Email: Maxim.Kosov@psi-cro.com

\begin{abstract}
The brain is a common site of tumor metastases, however patients with brain metastases are frequently either excluded from enrollment in clinical trials, or may be enrolled with limitations - clinically stable and/or previously treated. However, there is still no universally accepted definition of what to consider adequately treated and stable brain metastasis, which in turn, may lead to an excessively conservative approach to enroll such patients. During design of a study protocol that allows enrollment of patients with previously treated brain metastases, it is imperative to consider specifying the allowed previous treatment(s). We also recommend potential patients with a history of brain metastases to have a brain MRI as close to the planned start of chemotherapy, as possible, and if new lesions are identified, to treat them as appropriate before enrollment in the trial; this may give patients a chance to enter the trial, if the results of treatment are beneficial.
\end{abstract}

Keywords: Brain metastases; Imaging; Clinical trials; Enrollment; Gamma knife surgery; Inclusion and Exclusion criteria

Abbreviations: RCT: Randomized Clinical Trial; OS: Overall Survival; PFS: Progression-Free Survival; CNS: Central Nervous System; NSCLC: NonSmall Cell Lung Cancer; GKS: Gamma Knife Radio Surgery; IC: Informed Consent; MRI: Magnetic Resonance Imaging.

\section{Introduction}

Randomized clinical trials (RCT) are the "gold" standard of modern drug development. To assure comparability of different studies and unification of treatment response assessment different endpoints are being used. The main and possibly the only reliable goal of each late-phase cancer treatment is to achieve improvement of Overall Survival (OS), which is frequently used as the primary end-point. However, one characteristic of treatment efficacy and to some extent a surrogate marker of OS is Progression Free Survival (PFS) which is defined as "time from randomization until objective tumor progression or death" [1]. It is also a well-recognized endpoint and is widely used in oncology RCTs for solid tumors, especially in early-stage clinical trials.

The most frequently used tool for PFS assessment is RECIST 1.1criteria (response evaluation criteria in solid tumors) that were first published in 2000 and updated in 2009 [2]. At the same time, there is a growing evidence of RECIST limitations, especially in clinical trial setting. These limitations have been widely described in the literature and detailed discussion is beyond the scope of this article. However, one limitation is that all soft tissues/organs of the body are considered equal in terms of lesion measurements and response assessment. This is true when we speak about chest vs abdomen or pelvis. At the same time, there is one system that should be considered a specific one - central nervous system (CNS), and specifically - brain metastases of different malignancies. Routinely, RCTs either completely exclude enrolling patients with brain metastases, or put certain limitations. There are two main reasons for such an approach: lower survival rate in patients with brain metastases, which could masque efficacy of the study drug and lack of bloodbrain barrier penetration for some anticancer drugs.

The first statement was more true 15-20 years ago, when brain metastases were diagnosed at the late stages of cancer, when associated clinical signs had appeared. Modern diagnostic technologies allows in many cases identification of brain metastases while they are clinically silent, and thus, provide an opportunity of an early treatment with rather better survival. Excluding these patients is one of the major factors that limit enrollment in certain diseases and have serious impact on the patient population in the study vs "real life". For example, it is established that the incidence of brain metastases in advanced ALK-positive non-small cell lung cancer (NSCLC) ranges from 20 to $30 \%$ [3]. To overcome this, some protocols allow inclusion of 
patients with "previously treated with whole-brain radiotherapy or gamma-knife surgery and currently stable (or asymptomatic) brain metastases".

We searched clinicaltrials.gov to determine active studies in metastatic NSCLC and as of 20 September 2017, identified 197 interventional studies. Of these197 studies, 28(14\%) had strict exclusion criteria and did not allow enrollment of patient with known brain metastases, 58(30\%) allowed enrollment of patients with existing previously treated and stable brain metastases, 32(16\%) permitted enrollment of patients with asymptomatic brain metastases, regardless of their treatment, and $71(36 \%)$ did not mention brain involvement in inclusion/ exclusion criteria. The last group, where CNS metastases are not specified is difficult to interpret, as in the majority of cases, inclusion and exclusion criteria were not full with and additional criteria may be applicable. Interestingly, only 8(4\%) trials specifically required patients with brain metastases. Our data could be compared with the data in other malignances, for example, Tri Cao Le et al, in their recent search for CNS exclusion criteria in clinical trials in metastatic renal cell carcinoma, showed that $24 \%$ of studies strictly excluded patients with CNS metastases, which is slightly higher than in our search, and a comparable number of studies (57\%) enrolled patients with treated and/or stable brain metastases [4]. Thus, depending on the type of cancer, up to one fourth of patients may be excluded from participation in the clinical trials.

Another complicating issue is, that even if the protocol allows inclusion of patients with treated and controlled brain metastases, there is no universally accepted definition. Only a few protocols give specific options, e.g. treated with whole brain radiation or gamma knife radio surgery (GKS).Such uncertainties may lead to situations, when even patients with brain metastases that are allowed for enrollment may be taken off the study. To illustrate this statement, we present the following case from a clinical trial in NSCLC.

Patient with stage IV NCSCL with a history of brain metastases treated with GKS, was enrolled in a clinical trial. One week after the patient started treatment, and being asymptomatic, he underwent brain MRI, which was a follow up assessment after the previous evaluation, that was performed 6 weeks previously. This new MRI revealed two new small metastases in the right cerebellar hemisphere, which, in accordance with RECIST 1.1 criteria was considered progression of NSCLC. The investigator agreed that this was definitely progression based on the available imaging data. Nevertheless, the investigator argued that it was not possible to date the appearance of these metastases and in his opinion; they were present prior to the initiation of the study treatment. The patient was scheduled for GKS soon after the metastases were diagnosed, and the investigator asked for permission to continue the patient on study treatment. In clinical practice, it is rather common in such cases to treat brain metastases and resume chemotherapy. Considering that it was a study treatment, the decision was made to discontinue the patient from the clinical trial, as the study protocol requires considering any newly revealed metastasis as a disease progression. We also have to consider a possibility of post-radiosurgical peritumorous edema that may require administration of steroids, which is routinely used in clinical practice, but is frequently prohibited by clinical trial protocols $[5,6]$.

This case poses two important questions that in our opinion should be considered in planning clinical trials. First, routine brain imaging is not a part of radiology assessment during clinical trials, unless there were brain metastases prior to obtaining informed consent (IC). In this patient, the investigator decided not to perform brain MRI at screening, as the previous one, was done two weeks prior to signing Informed Consent. Screening per protocol last to up to 28 days, and this resulted in an almost 6 week interval between the brain MRI and initiation of therapy. Repeat MRI was scheduled one week after the initiation of therapy, so it seems reasonable to shift it to an earlier date, prior to randomization. Even if new metastases are seen (which makes the patient ineligible), there was an option to perform GKS and having done this in case of a good result, the patient could after due time fit the protocol criterion of "stable, previously treated brain metastases).

Second, is there any difference in outcome if GKS is performed prior to the start of chemotherapy, or shortly after its initiation? To our knowledge, based on the literature, no difference in outcome was reported. But this question has more practical than research aspect at the moment.

\section{Conclusion}

Our suggestions are: when a study protocol allows enrollment of patients with previously treated brain metastases - to specify the preferable, or the only allowed treatment(s), and if a potential patient has had brain metastases, we recommend to perform brain MRI as close to the planned start of chemotherapy, as possible, and if new lesions are identified, to treat them with GKS (or as appropriate)before enrollment in the trial, what could grant patients a chance to enter the trial if the results of this treatment are beneficial.

We also think that in some clearly defined situations study treatment could be interrupted and resumed after successful treatment of newly revealed brain metastases. However, the latter suggestion at the moment is a bit provocative and needs deep thinking over and discussion.

\section{References}

1. Food and Drug Administration. Guidance for Industry: Clinical Trial Endpoints for the Approval of Cancer Drugs and Biologics.

2. Eisenhauer EA, Therasse P, Bogaerts J, Schwartz LH, Sargent D, et al. (2009) New response evaluation criteria in solid tumors: revised RECIST guideline (version 1.1). Eur J Cancer 45(2): 228-247. 
3. Solomon BJ, Mok T, Kim DW, Wu YL, Nakagawa K, et al. (2014) First-line crizotinib versus chemotherapy in ALK-positive lung cancer. N Engl J Med 371(23): 2167-2177.

4. Tri Cao Le, Brugarolas J (2017) The enrollment status of patients with brain metastases in metastatic renal cell carcinoma: a query of clinicaltrials gov. J Clin Oncology 35 (suppl): e16070
5. Takakura K, Hayashi M, Chernov MF, Tamura N, Izawa M, et al. (2013) Gamma knife treatment strategy for metastatic brain tumors. Acta Neurochir 116(Suppl): 63-69.

6. Chernov MF, Hayashi M, Ganz JC, Takakura K (2013) Gamma knife neurosurgery in the management of intracranial disorders.

\begin{tabular}{|l|}
\hline \multicolumn{1}{|c|}{ Your next submission with Juniper Publishers } \\
will reach you the below assets \\
- Quality Editorial service \\
- Swift Peer Review \\
- Reprints availability \\
- E-prints Service \\
- Manuscript Podcast for convenient understanding \\
- Global attainment for your research \\
- Manuscript accessibility in different formats \\
( Pdf, E-pub, Full Text, Audio) \\
- Unceasing customer service \\
Track the below URL for one-step submission \\
https://juniperpublishers.com/online-submission.php \\
\hline
\end{tabular}

